Proceedings

\title{
A New Type of LSPR Sensor Featuring Immobilized Liposome or Phospholipid Single Layer ${ }^{\dagger}$
}

\author{
Masahiro Kawasaki ${ }^{1}$, Hiroyuki Yoshikawa ${ }^{2}$, Masato Saito ${ }^{2}$, Shu Jiang ${ }^{2}$, Takehiro Akeyama ${ }^{2}$, \\ Eiichi Tamiya ${ }^{2}$ and Minoru Noda ${ }^{1, *}$ \\ 1 Electronics, Kyoto Institute of Technology, Kyoto 606-8585, Japan; mhks.smys@gmail.com \\ 2 Graduate School of Engineering, Osaka University, Suita 565-0871, Japan; \\ yosikawa@ap.eng.osaka-u.ac.jp (H.Y.); saitomasato@ap.eng.osaka-u.ac.jp (M.S.); \\ kyoujo@ap.eng.osaka-u.ac.jp (S.J.); akeyama@ap.eng.osaka-u.ac.jp (T.A.); \\ tamiya@ap.eng.osaka-u.ac.jp (E.T.) \\ * Correspondence: noda@kit.ac.jp; Tel.: +81-75-724-7443 \\ † Presented at the Eurosensors 2018 Conference, Graz, Austria, 9-12 September 2018. \\ Published: 4 December 2018
}

\begin{abstract}
We have fabricated a new type of LSPR sensor featuring immobilized liposome or phospholipid single layer. LSPR principally shows an ultrahigh sensitivity on surface dielectric environmental change due to interaction with target, but little has been reported so far on applying phospholipid membranes and/or liposomes as model cell membrane. We newly tried to investigate biosensing capabilities using the membranes of the both structures on Au nanostructures of LSPR sensor chip. As a result, it was confirmed that the phospholipid single layer is more effective to improve the sensitivity than the liposome. Finally, we have clearly detected $100 \mathrm{nM}$ target protein of $\mathrm{CAB}$ and estimated a possible detection of $10 \mathrm{nM}$ range from wavelength resolution by interaction with the phospholipid single layer.
\end{abstract}

Keywords: LSPR; biosensor; nanostructure; nanopillar; phospholipid; liposome; high-sensitive detection

\section{Introduction}

As for one of the reasons why the application of phospholipid and its effective performance on Localized Surface Plasmon Resonance (LSPR) sensing have been little shown so far, we consider that the phenomena of near-field light would not be so strong in the interaction on the membrane immobilized on $\mathrm{Au}$ nanostructure, compared to the case in antigen-antibody reaction occurring directly on Au surface.

We have fabricated a new type of LSPR sensor featuring immobilized liposome or phospholipid single layer. LSPR principally shows an ultrahigh sensitivity on surface dielectric environmental change due to interaction with target, but little has been reported so far on applying phospholipid membranes [1] and/or liposomes as model cell membrane, although the phospholipid membrane has a variety of attractive capabilities to detect target molecular dynamics. We newly tried to investigate biosensing capabilities using the membranes of the both structures on Au nanostructures of LSPR sensor chip. As a result, it was confirmed that the phospholipid single layer is more effective to improve the sensitivity than the liposome. Finally, we have clearly detected $100 \mathrm{nM}$ target protein of $\mathrm{CAB}$ and estimated a possible detection of $10 \mathrm{nM}$ range from wavelength resolution by interaction with the phospholipid singlelayer. 


\section{Materials and Methods}

For the Au nanostructures, we used gold-capped nanopillars imprinted thermally on a polymer film, which was developed previously by one of our authors [2]. The polymer is Cyclo-olefin polymer (COP). The fabrication steps of the structure is illustrated in Figure 1; (a) fabrication of porous alumina mold, (b) thermal nanoimprinting with high temperature and pressure to the COP resin. (c) formation of the nanopillar structure by releasing from the alumina porous mold, and (d) final Au-sputtered and capped nanopillar structures for the LSPR substrate. Figure 2 shows an SEM view of fabricated Au nanopillars. It is observed that designed structures are successfully formed.

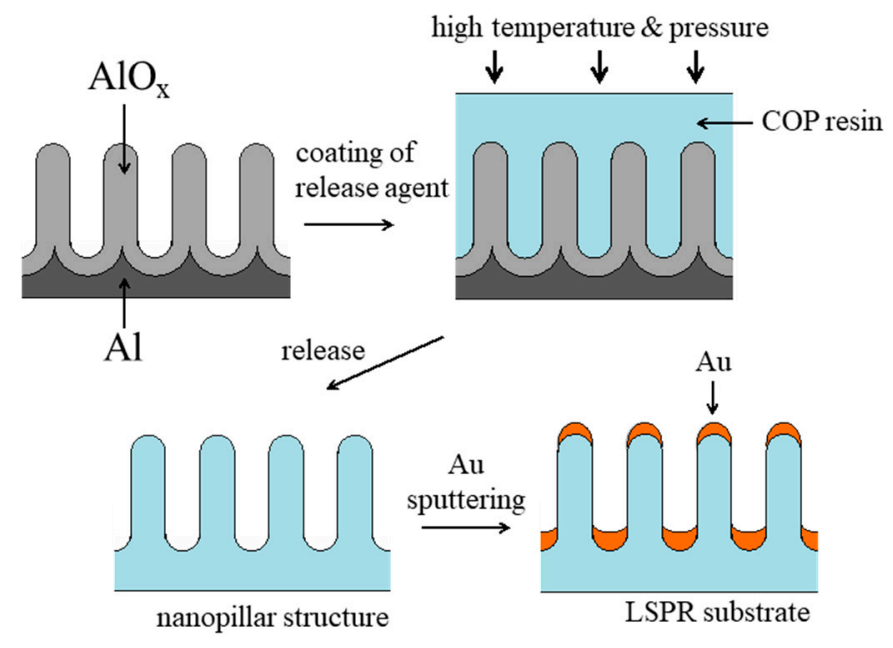

Figure 1. The used fabrication steps of the Au nanopillar structure [2].

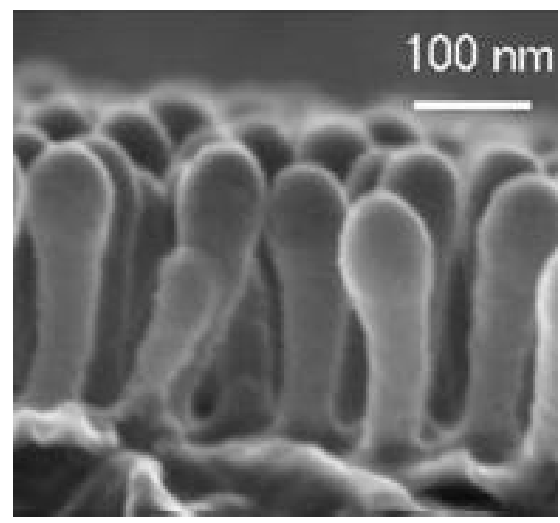

Figure 2. An SEM view of fabricated Au nanopillars [2].

Figure 3 show cross-sectional illustrations of LSPR sensor immobilized with (a) liposomes and (b) phospholipid single layer. Au island was formed on the tip of nanopillar. DMPC liposomes or its single-layered phospholipids were immobilized on the nanostructures. Carbonic anhydrase from bovine ( $\mathrm{CAB}$ ) was used as a target protein because its interaction was confirmed with some different phospholipids.

Figure 4 shows our LSPR measurement system. Absorption spectra of nanopillar films were measured with a spectroscopy system consisting of a spectrophotometer (USB-4000-UV-vis), a tungsten halogen light source (LS-1), optical fibers (R-400-7 UV/vis, fiber core diameter: $200 \mu \mathrm{m}$ ), and collimating lenses (74-UV), all purchased from Ocean Optics. Nanopillar films immobilized on a glass microscope slide with carbon tapes were put on the sample stage of the spectroscopy system. 

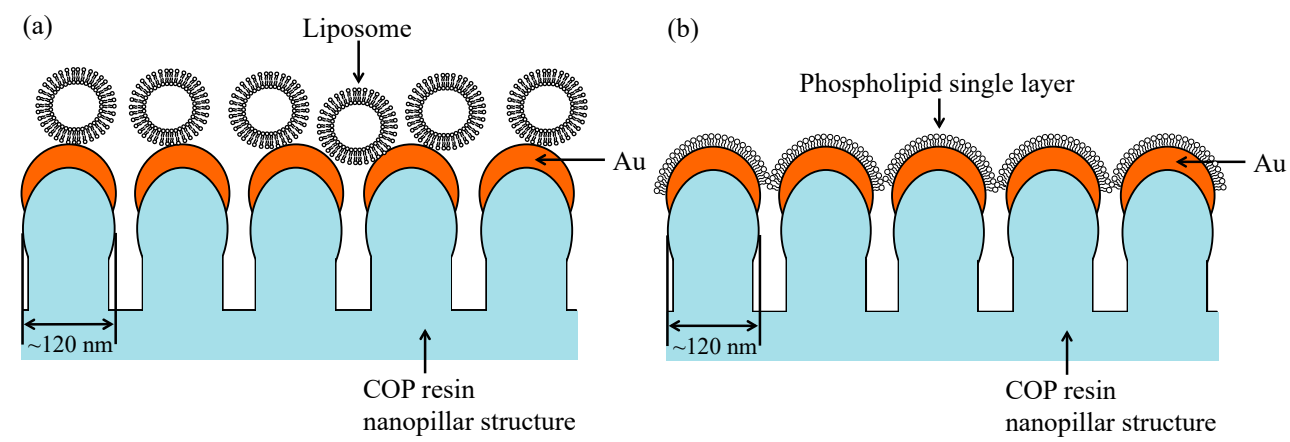

Figures 3. Cross-sectional illustrations of LSPR sensor immobilized with (a) liposomes and (b) phospholipid single layer.

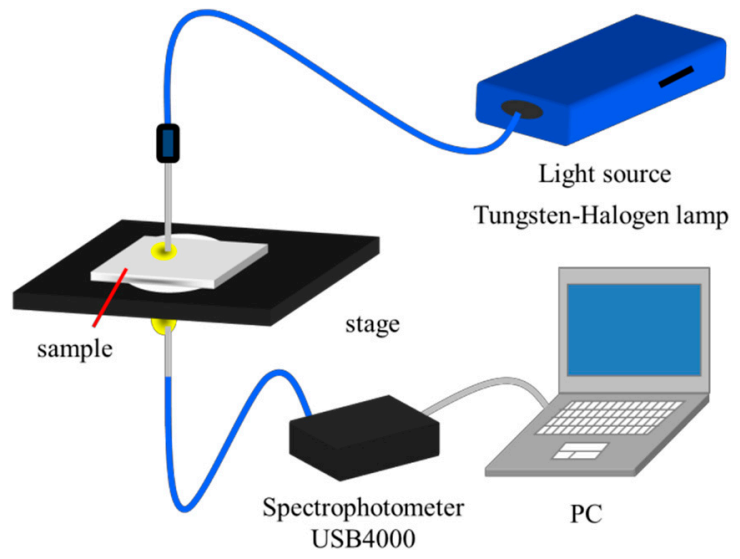

Figure 4. LSPR measurement system.

\section{Results and Discussion}

As shown in Figure 5, absorption spectrum of a bare Au/nanopillar shows maximum peak corresponding to LSPR of Au nanostructures at $\sim 540 \mathrm{~nm}$. With immobilized liposome, LSPR peak wavelength shifts showed continuously positive increase from bare Au surface, after SAM deposition, immobilization of liposome, and finally after addition of $10 \mu \mathrm{M} \mathrm{CAB}$. But the sensitivity would degrade as the size of liposome (about $100 \mathrm{~nm}$ ) was larger than the range of near-field effect (a few to tens of $\mathrm{nm}$ ) from the Au nanostructure. From Figure 5, the characteristic peak wavelength also shows clearly a monotonous shift from bare Au surface, after SAM deposition, immobilization of DMPC single layer, and finally after addition of $10 \mu \mathrm{M} \mathrm{CAB}$. The sensitivity is expected to improve more for the membrane, because its size (thickness) is around $3 \mathrm{~nm}$ and within the range of the near-field effect. After decreasing the target $\mathrm{CAB}$ concentration, finally, Figure 6 suggests that $100 \mathrm{nM} C \mathrm{CAB}$ was clearly detected and the sensitivity improved by about 2 orders of magnitude from the case with the liposome.

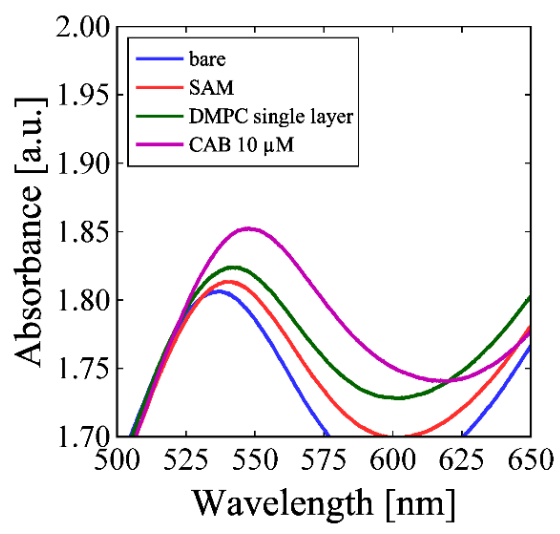

Figure 5. Absorbance for immobilized with DMPC single layer vs LSPR wavelength. 


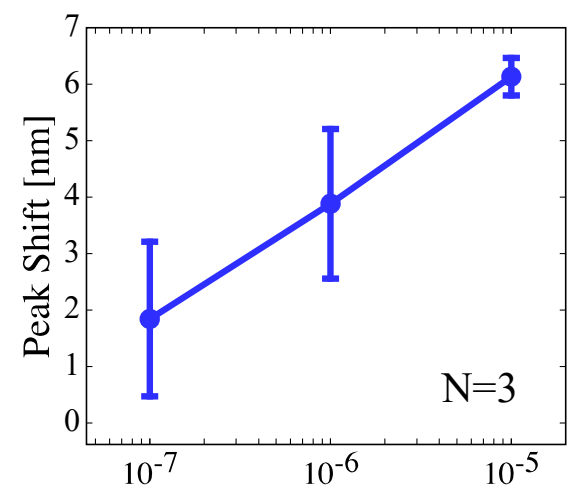

CAB Protein Concentration $[\mathrm{mol} / \mathrm{L}]$

Figure 6. LSPR peak wavelength shift vs target $\mathrm{CAB}$ protein concentration.

\section{Conclusions}

We have fabricated a new type of LSPR sensor featuring immobilized liposome or phospholipid single layer. By the sensor, we tried to investigate biosensing capabilities using the membranes of the both structures on Au nanostructures of LSPR sensor chip. As a result, a monotonous increase in the shift of LSPR peak wavelength was successfully observed depending on successive formation on the $\mathrm{Au}$ nanopillars of SAM layer, immobilized liposome or phospholipid single layer and interacted target protein. Thereafter, it was confirmed that the phospholipid single layer is more effective to improve the sensitivity than the liposome. Finally, we have clearly detected $100 \mathrm{nM}$ target protein of $\mathrm{CAB}$ and estimated a possible detection of $10 \mathrm{nM}$ range from wavelength resolution by interaction with the phospholipid single layer.

\section{References}

1. Hiep, H.M.; Endo, T.; Saito, M.; Chikae, M.; Kim, D.K.; Yamamura, S.; Takamura, Y.; Tamiya, E. Label-Free Detection of Melittin Binding to a Membrane Using Electrochemical-Localized Surface Plasmon Resonance. Anal. Chem. 2008, 80, 1859-1864.

2. Saito, M.; Kitamura, A.; Murahashi, M.; Yamanaka, K.; Hoa le, Q.; Yamaguchi, Y.; Tamiya, E. Novel Gold-Capped Nanopillars Imprinted on a Polymer Film for Highly Sensitive Plasmonic Biosensing. Anal. Chem. 2012, 84, 5494-5500.

(C) 2018 by the authors. Licensee MDPI, Basel, Switzerland. This article is an open access article distributed under the terms and conditions of the Creative Commons Attribution (CC BY) license (http://creativecommons.org/licenses/by/4.0/). 